\title{
Campanha nacional de detecção de casos suspeitos de diabetes mellitus no Brasil: relatório preliminar ${ }^{1}$
}

\author{
Romero Bezerra Barbosa, ${ }^{2}$ Alberto Barceló ${ }^{3}$ \\ e Carlos Alberto Machado 4
}

RESUMO Para reduzir a morbi-mortalidade associada ao diabetes, o Ministério da Saúde do Brasil está implantando o Plano de Reorganização da Atenção à Hipertensão Arterial e ao Diabetes Mellitus. Como parte desse plano (composto de quatro etapas), realizou-se, em março e abril de 2001, a campanha nacional de detecção de casos suspeitos de diabetes, o primeiro levantamento desse tipo realizado pelos serviços públicos de saúde no Brasil. Os resultados preliminares são descritos no presente artigo. De um total de 5507 municípios participantes, 4446 (81\%) enviaram dados ao Ministério da Saúde. Foram testados 20 milhões de pessoas (71\% da população-alvo), identificando-se 3,3 milhões de suspeitos de diabetes. A campanha contribuirá para a reestruturação do atendimento sistemático e resolutivo aos portadores de diabetes no Sistema Único de Saúde. Os resultados preliminares confirmam o diabetes como um dos principais problemas de saúde no Brasil. A prevenção de diabetes tipo 2 deve receber prioridade.

Palabras clave Triagem, administração de serviços de saúde

As doenças do aparelho circulatório representam um importante problema de saúde pública no Brasil. Desde a dé- cada de 1960, as doenças cardiovasculares são a primeira causa de morte nesse país, segundo os registros ofi-

\footnotetext{
1 Esta campanha é uma etapa do Plano de Reorganização da Atenção à Hipertensão Arterial e ao Diabetes Mellitus. O Comitê Técnico Nacional do Plano de Reorganização da Atenção à Hipertensão Arterial e ao Diabetes Mellitus é constituído por: Carlos Alberto Machado, Secretaria de Políticas de Saúde (SPS), Ministério da Saúde; Carlos Alberto Pereira Gomes, Área de Assistência Farmacêutica (SPS), Ministério da Saúde; Juliana Ferraz, Secretaria de Assistência à Saúde (SAS), Ministério da Saúde; Celso Amodeo, Sociedade Brasileira de Cardiologia/Fundação do Coração (FUNCOR); José Marcio Ribeiro, Sociedade Brasileira de Cardiologia/Departamento de Hipertensão Arterial; Osvaldo Kohlmann Junior, Sociedade Brasileira de Hipertensão; Adriana Costa Forti, Sociedade Brasileira de Diabetes; José Nery Praxedes, Sociedade Brasileira de Nefrologia, Departamento de Hipertensão Arterial; Fadlo Fraige Filho, Federação Nacional de Associações de Diabéticos; Maria Acioly Mota, Federação Nacional de
}

Associações de Hipertensão Arterial; Alcy Moreira dos Santos Pereira, Conselho Nacional de Secretários Estaduais de Saúde (CONASS); Valter Luiz Lavinas Ribeiro, Conselho Nacional de Secretários Municipais de Saúde (CONASEMS).

2 Ministério da Saúde, Secretaria de Políticas de Saúde, Área Técnica de Hipertensão Arterial e Diabetes Mellitus. Correspondência e pedidos de separatas devem ser enviados a este autor no seguinte endereço: Área Técnica de Hipertensão Arterial e Diabetes Mellitus, SPS, Ministério da Saúde, Esplanada dos Ministérios, Bloco 11, sexto andar, CEP 70058-900, Brasília, DF, Brasil. Telefone: +55-61225-6388; fax: +55-61-315-2848; e-mail: romero. bezerra@saude.gov.br

3 Organização Pan-Americana da Saúde.

4 Ministério da Saúde, Secretaria de Políticas de Saúde, Coordenação do Plano de Reorganização da Atenção à Hipertensão Arterial e ao Diabetes Mellitus. ciais do Sistema de Informação sobre Mortalidade (SIM) (figura 1). Em 1998, corresponderam a mais de $25 \%$ do total de óbitos.

Por sua vez, o diabetes mellitus (DM) constitui-se em um dos principais fatores de risco para as doenças do aparelho circulatório. Entre as conseqüências mais freqüentes do DM encontram-se o infarto agudo do miocárdio (IAM), o acidente vascular encefálico (AVE), a insuficiência renal crônica, as amputações de pés e pernas, a cegueira definitiva, os abortos e as mortes perinatais. Dados de um estudo sobre o DM no Brasil, realizado em 1987 pelo Ministério da Saúde, Sociedade Brasileira de Diabetes, Conselho Nacional de Desenvolvimento 
FIGURA 1. Mortalidade proporcional segundo grupos de causas selecionadas em capitais, Brasil, 1930 a 1994

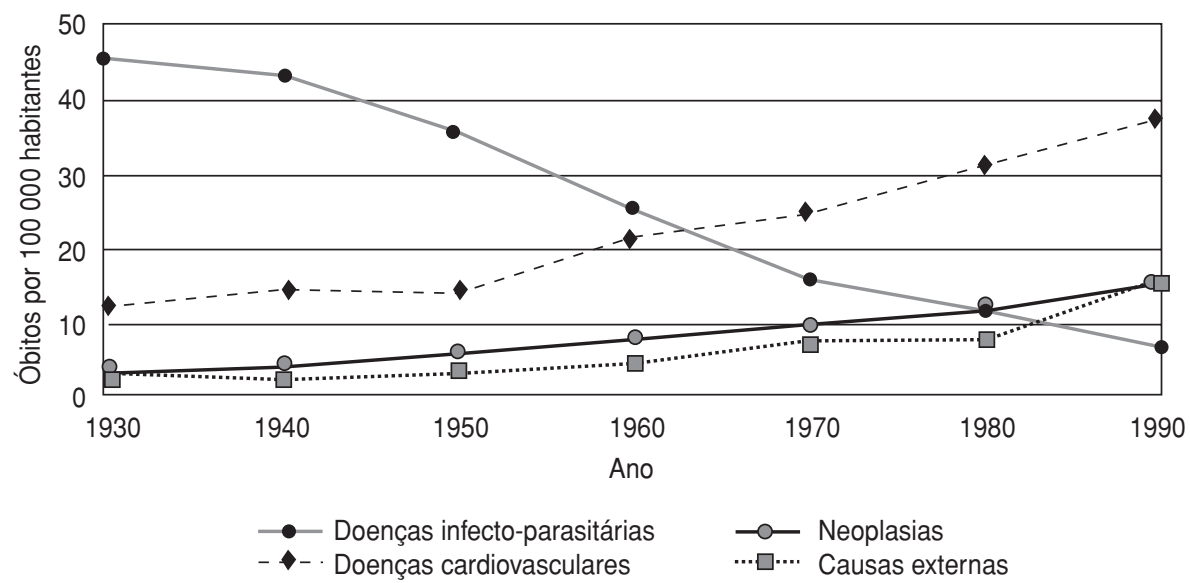

Fonte: Bayer e Paula (1). Excluídas as causas mal definidas.

Científico e Tecnológico (CNPq) e outros, demonstraram uma prevalência de $8 \%$ na população de 30 a 69 anos de idade (2). O estudo revelou também um alto grau de desconhecimento em relação à doença. Cerca de $50 \%$ dos diagnosticados não suspeitavam de que tinham diabetes.

Outros estudos realizados na América Latina mostraram não só que $50 \%$ dos casos novos de diabetes já tinham complicações crônicas no momento do diagnóstico, como também que aproximadamente metade das complicações detectadas estavam em estágio avançado. Tais resultados falam em favor da identificação precoce dos casos de diabetes como forma de oportunizar a prevenção de suas graves complicações. Informações provenientes de estudos randomizados sobre o diabetes tipo 1 e o diabetes tipo 2 indicam claramente a redução da ocorrência de complicações por meio do controle metabólico da doença $(3,4)$.

Além disso, a identificação oportuna de casos favorece o estabelecimento de vínculos entre os pacientes e as unidades básicas de saúde, o que é imprescindível para o sucesso do controle da doença. Entre as vantagens do acompanhamento e controle do DM no âmbito da atenção básica estão a possibili- dade de evitar o surgimento de complicações, assim como de evitar o agravamento destas ocorrências, reduzindo tanto o número de internações hospitalares quanto a mortalidade relacionada às doenças cardiovasculares.

No Brasil, apesar das várias experiências municipais bem sucedidas de acompanhamento dos casos de DM, observa-se, em grande parte do país, a falta de vínculos entre os pacientes e as unidades de saúde. Em regra, o atendimento aos pacientes ocorre de modo assistemático, nos serviços de emergência, espaço esse que não propicia nem a identificação de lesões em órgãos-alvo do DM nem o estabelecimento de um programa de tratamento e acompanhamento adequado a cada caso. No que se refere à promoção da saúde e à prevenção dos fatores de risco, a situação é ainda mais crítica, tendo em conta a falta de preparação e de tradição dos serviços de saúde para a realização sistemática de tais atividades.

Com o propósito de contribuir para a redução da morbi-mortalidade associada ao DM e à hipertensão arterial, o Ministério da Saúde do Brasil, as secretarias estaduais e municipais de saúde, as sociedades científicas de diabetes, cardiologia, hipertensão arterial e nefrologia, a Federação Nacional de
Associações de Diabéticos e Hipertensão e os Conselhos Nacionais de Secretários Estaduais e Municipais de Saúde assumiram o compromisso de empreender a reorganização da rede de saúde. Dessa forma, está sendo implantado, em todo o país, o Plano de Reorganização da Atenção à Hipertensão Arterial e ao Diabetes Mellitus (5). Tal plano visa, entre outros, melhorar a atenção às pessoas com diabetes e hipertensão arterial mediante o desenvolvimento de ações articuladas de promoção da saúde, prevenção, tratamento e controle dessas doenças.

A campanha nacional, uma das quatro etapas do plano, realizada em março e abril de 2001, é o primeiro levantamento para detecção de suspeitos de DM realizado pelos serviços públicos de saúde no Brasil. É, também, o primeiro passo para a mencionada reorganização do atendimento aos pacientes. Embora sejam conhecidas pesquisas clínicas para detecção do DM realizadas na Inglaterra e nos Estados Unidos (6), não existe na literatura relato de nenhuma experiência nacional, com testagem maciça da população, utilizando o sistema público de saúde, o que faz aumentar o interesse com relação à campanha realizada no Brasil.

O objetivo geral da campanha nacional brasileira foi identificar e vincular às equipes da rede de serviços básicos de saúde, em 5500 municípios brasileiros, os portadores de DM que utilizam o Sistema Único de Saúde (SUS). Os objetivos específicos da campanha foram:

- detectar suspeitos de DM entre os usuários do SUS, por meio do teste de glicemia capilar;

- apoiar as secretarias municipais de saúde no cadastramento e vinculação dos portadores de DM à rede de serviços básicos de saúde;

- atualizar os profissionais da rede de serviços básicos e os profissionais do programa de saúde da família (PSF) em termos do atendimento e acompanhamento dos pacientes, com base em protocolos clínicos padronizados;

- melhorar a oferta de medicamentos para o tratamento do DM; 
- ampliar o conhecimento sobre a freqüência e distribuição do DM no Brasil.

A população alvo foi constituída por usuários do SUS com idade igual ou superior a 40 anos, totalizando um contingente de 31239703 indivíduos, segundo estimativa do Instituto Brasileiro de Geografia e Estatística (IBGE).

A campanha nacional de detecção de suspeitos de DM foi realizada de 6 de março a 7 de abril de 2001. Para a realização dessa etapa, o Ministério da Saúde forneceu aos municípios 19500 glicosímetros e 37,7 milhões de tiras reagentes e lancetas. Os aparelhos foram utilizados para efetuar testes de glicemia capilar de acordo com a técnica de leitura comparativa ou óptica através das tiras reagentes. Esse teste é aprovado pela agência Food and Drug Administration (FDA), dos Estados Unidos, e possui um grau de confiabilidade superior a $90 \%$ quando comparado com a glicemia plasmática. Às empresas fornecedoras dos glicosímetros coube a responsabilidade do treinamento dos profissionais da rede assistencial.

Foram considerados suspeitos os indivíduos que apresentaram glicemia em jejum com valores iguais ou superiores a $100 \mathrm{mg} / \mathrm{dL}$ e glicemia pós-prandial com valores maiores do que $140 \mathrm{mg} / \mathrm{dL}$.

O Ministério da Saúde produziu e distribuiu 100000 cartazes de divulgação da campanha nos estabelecimentos de saúde, 200000 cartazes para convocação e esclarecimento da população, 2 milhões de folhetos informativos e 25000 cartazes de mesa contendo orientações para os profissionais da rede de serviços. O material impresso foi entregue aos municípios comprometidos com a realização da campanha. As atividades de divulgação e mobilização da população foram planejadas e implementadas pela assessoria de comunicação social

TABELA 1. Campanha nacional, Plano de Reorganização da Atenção à Hipertensão Arterial e ao Diabetes Mellitus, Brasil, 2001

\begin{tabular}{lcccc}
\hline & População & Número de exames & \multicolumn{2}{c}{ Suspeitos } \\
\cline { 5 - 5 } \multicolumn{1}{c}{ Região } & \multicolumn{1}{c}{ realizados } & 1127483 & Número & $\%$ \\
\hline Norte & 1676061 & 5791026 & 157410 & 14 \\
Nordeste & 8159089 & 1526702 & 935917 & 16 \\
Centro-oeste & 1862473 & 9250833 & 1391317 & 17 \\
Sudeste & 14503011 & 3129681 & 583684 & 15 \\
Sul & 5039069 & 20825726 & 3322576 & 16 \\
Brasil & 31239703 & &
\end{tabular}

do Ministério da Saúde, com a inserção de mensagens em redes nacionais de TV, outdoors, jornais e revistas de grande circulação.

De um total de 5507 municípios participantes, 4446 (81\%) enviaram dados ao Ministério da Saúde no período aprazado. A população-alvo dos municípios informantes corresponde a 28,16 milhões de pessoas (tabela 1). Nesses municípios, foram testados 20 milhões de pessoas, ou seja, $71 \%$ do total da população-alvo, identificando-se 3,3 milhões de suspeitos de $\mathrm{DM}$, o que corresponde a $16 \%$ do total da população testada. Com base em dados de prevalência encontrados em estudo regional anterior (2), poderiam ser esperados 2,52 milhões de diabéticos na população-alvo da campanha. Os suspeitos foram agendados para testes de confirmação com dosagem da glicemia plasmática.

Como forma de assegurar que os achados positivos da etapa de detecção de suspeitos recebessem tratamento com esquema terapêutico padronizado, o Ministério da Saúde realizou a aquisição e distribuição dos hipoglicemiantes glibenclamida $5 \mathrm{mg}$ e metformina, totalizando um gasto de cerca de 13,5 milhões de reais (o que equivale, atualmente, a aproximadamente 4,9 milhões de dólares); 172 milhões de comprimidos de glibenclamida $5 \mathrm{mg}$ foram entregues aos serviços de saúde dos municípios participantes, o que corresponde a uma cobertura de mais de 1,7 milhões de pessoas afetadas pelo DM em uso de medicação por 6 meses.

Além do benefício de detectar novos casos, a campanha nacional contribuiu para a estruturação do atendimento sistemático aos portadores de DM no SUS, na medida que deu início ao cadastramento e vinculação dos pacientes às unidades básicas da rede de serviços. Como efeito posterior, espera-se que a ampla capacitação de recursos humanos efetivada para levar a cabo a campanha venha a melhorar o acompanhamento desses pacientes e, conseqüentemente, a sua adesão ao tratamento adequado.

Em conclusão, a campanha nacional de detecção de diabetes foi conduzida com êxito e obteve uma participação importante da população. Os resultados preliminares confirmaram que o diabetes é um dos principais problemas de saúde no Brasil. A prevenção de diabetes tipo 2 deve ser considerada uma prioridade de saúde pública. 


\section{REFERÊNCIAS}

1. Bayer GF, Paula SG. Mortalidade nas capitais brasileiras 1930-1980. Radis/FIOCRUZ. Dados 1984;7:1-8.

2. Brasil, Ministério da Saúde. Estudo multicêntrico sobre a prevalência do DM no Brasil: resultados. Brasília: Ministério da Saúde; 1991

3. Distrito Federal, Secretaria de Saúde, Hospital Regional de Taguatinga. Projeto salvando o pé do diabético: programa de educação e controle de diabetes. Brasília: Secretaria de Saúde do Distrito Federal; 1992.

4. Boulton AJ, Gries FA, Jervell JA. Guidelines for the diagnosis and outpatient management of diabetic peripheral neuropathy. Diabet Med 1998;15(6):508-514.

5. Brasil, Ministério da Saúde, Secretaria de Políticas de Saúde. Plano Nacional de Reorganização da Atenção à Hipertensão Arterial e ao Diabetes Mellitus. Brasília: Ministério da Saúde; 2001.
6. American Diabetes Association. Clinical practice recommendation 2000. Diabetes Care 23(suppl 1);2000.

Manuscrito recebido em 6 de setembro de 2001. Aceito em 21 de setembro de 2001 .

ABSTRACT The Ministry of Health of Brazil is implementing a National Plan for the Reorganization of Health Care for Hypertension and Diabetes Mellitus, with the aim of reducing the morbidity and mortality associated with diabetes. This plan is divided into four stages, one of which was a national campaign to detect suspected diabetes cases that was carried out in March and April 2001. This was the first survey of its kind carried out by public health services in Brazil, and the preliminary results are described in this article. Out of a total of 5507 participating municipalities, 4446 of them (81\%) submitted data to the Ministry of Health. Twenty million people were tested $(71 \%$ of the target population), and 3.3 million of them were identified as possibly having diabetes. The campaign will contribute towards restructuring the systematic and resolution-oriented care that the national, public Unified Health System provides to diabetics. The preliminary results confirm that diabetes is one of the main health problems in Brazil. Priority should be given to preventing type 2 diabetes.

\section{National campaign to detect suspected diabetes cases in Brazil: a preliminary report}

\section{Society of Behavioral Medicine Annual Meeting}

\author{
Dates: $\quad$ 3-6 April 2002 \\ Location: Omni Shoreham Hotel \\ Washington, D.C., United States of America
}

The theme of the 2002 Annual Meeting of the Society of Behavioral Medicine (SBM) is "beyond adherence: building partnerships among individuals, clinicians, and systems."

The meeting will include keynote speeches, master lectures, paper presentations, poster presentations, symposia, and other events. Among the conference topics will be addictive behaviors and alcohol and tobacco abuse, chronic disease management, population health and health policy, prevention of risk behaviors, psychosocial influences on disease, and translating research into practice.

The registration fee for the conference is US\$ 185 for SBM members and US\$ 335 for others.

\section{Information:}

The Society of Behavioral Medicine

7600 Terrace Avenue, Suite 203

Middleton, Wisconsin 53562, United States of America

Telephone: (608) 827-7267

Fax: (608) 831-5485

E-mail: info@sbmweb.org

World Wide Web site: http://www.sbmweb.org 